\title{
Metástasis en cuero cabelludo como primera manifestación de cáncer de tiroides mixto folicular-papilar
}

\author{
Scalp metastasis as the first manifestation of mixed follicular- \\ papillary thyroid cancer
}

\author{
S. Junquera Bañares ${ }^{1}$, E. Oria Mundín ${ }^{1}$, T. Tuñón Álvarez ${ }^{2}$, C. Vázquez Martínez ${ }^{3}$ \\ J.I. Botella Carretero ${ }^{3}$
}

\section{RESUMEN}

La aparición de metástasis a distancia en los cánceres diferenciados de tiroides es poco frecuente, y cuando se detectan en piel, suelen darse en el contexto de una enfermedad diseminada después de muchos años de evolución desde el diagnóstico. Se presenta el caso de una paciente de 77 años, sin enfermedad tiroidea conocida que debutó con una metástasis en región parietooccipital del cuero cabelludo en la que se identificó tejido tiroideo. En la ecografía tiroidea se identificó un nódulo informado como tumor folicular y el estudio de extensión precirugía fue negativo. Se le hizo tiroidectomía total más vaciamiento ganglionar del compartimento central y el estudio AP fue informado de carcinoma mixto folicular-papilar sin afectación ganglionar. En el RCT post-I131 (dosis de 104,7 mCi) se detectaron micrometástasis pulmonares, y a los 2 meses de una segunda dosis de I131 (125 mCi), la tiroglobulina fue indetectable.

El cáncer tiroideo debe incluirse en el diagnóstico diferencial de las metástasis cutáneas, puesto que su diagnóstico y tratamiento temprano, excepto en los casos de enfermedad muy avanzada, el pronóstico de su hallazgo suele ser más favorable que en el resto de tumores sólidos.

Palabras clave. Cáncer tiroides mixto folicular-papilar. Metástasis. Piel.

\begin{abstract}
Distant metastases are a rare occurrence in differentiated thyroid cancer, and when detected in skin, often arise in the context of disseminated disease after many years of progression since diagnosis. This study presents the case of a 77-year-old female without known thyroid disease, who presented with metastases in the parieto-occipital scalp region, in which thyroid tissue was identified. Thyroid ultrasound identified a nodule reported as a follicular tumour and preoperative investigation of tumour spread was negative. Total thyroidectomy plus central lymph node dissection were performed and histopathology reported mixed papillary-follicular carcinoma without lymph node metastasis. Lung micrometastases were detected in the post-I131 whole-body scan (104.7 $\mathrm{mCi}$ dose), and two months after a second dose of $131 \mathrm{I}(125 \mathrm{mCi})$, thyroglobulin was undetectable.
\end{abstract}

Thyroid cancer should be included in the differential diagnosis of cutaneous metastases, since with early diagnosis and treatment, prognosis is generally more favourable than in other solid tumours.

Keywords. Mixed papillary-follicular thyroid cancer Metastasis. Skin.
1. Servicio de Endocrinología y Nutrición. Complejo Hospitalario de Navarra.

2. Servicio de Anatomía Patológica. Complejo Hospitalario de Navarra.

3. Servicio de Endocrinología y Nutrición. Hospital Ramón y Cajal. Madrid.

\section{Correspondencia:}

S. Junquera Bañares

Servicio de Endocrinología y Nutrición

Complejo Hospitalario de Navarra

Irunlarrea, 3

31008 Pamplona

E-mail: sojuba@hotmail.com 


\section{INTRODUCCIÓN}

El cáncer de tiroides supone el 1\% de todos los cánceres y existen varios tipos: papilar, folicular, de células de Hürtle, medular, insular (pobremente diferenciado) y anaplásico. El carcinoma papilar es el más frecuente, se da en el 50-89\% de los casos y tiene una progresión lenta. Aparecen metástasis a distancia en menos del 10\% de los pacientes, y en un $4 \%$ se anteponen al diagnóstico del primario, y en menos del $1 \%$ se dan en piel. Los sitios más frecuentes de metastatización son los nódulos linfáticos cervicales (40\% de los casos), metástasis pulmonares (49\%), hueso (25\%), pulmón y hueso (15\%) y SNC $(12 \%)^{1}$. El pronóstico es muy bueno con una supervivencia a los 10 años del 80-90\%, incluso en los pacientes jóvenes con metástasis en nódulos linfáticos y/o pulmón al diagnóstico ${ }^{2}$.

El carcinoma folicular aparece el segundo en frecuencia (10-20\% de los casos), pero es el primero de los tipos diferenciados en producir metástasis a distancia, observándose en el 11-20\% de los casos. Curiosamente, aunque el cáncer folicular constituye sólo el $15 \%$ de los carcinomas diferenciados de tiroides (excluyendo medular y anaplásico), el $42 \%$ de los casos con metástasis cutáneas son debidas a este tipo de tumor ${ }^{3}$.

La incidencia de metástasis cutáneas de cáncer de tiroides es muy baja y suele verse en el contexto de una enfermedad diseminada después de años del diagnóstico.

Se expone el caso de una paciente sin antecedentes de patología tiroidea que presenta una metástasis en cuero cabelludo como primera manifestación de un cáncer mixto folicular-papilar de tiroides.

\section{CASO CLÍNICO}

Mujer de 77 años sin antecedentes de interés que consultó por una lesión en cuero cabelludo de más de dos años de evolución con crecimiento progresivo en el último año y que le molestaba con el roce. Se trataba de una lesión papulosa eritemato-violácea dura de $6 \mathrm{~mm}$ en región parietooccipital (Fig. 1a). Esta lesión se le extirpó mediante rebanado y nitrato de plata, sangró mucho y no se pudo obtener muestra suficien- te para estudio anatomopatológico (AP). A los 6 meses le volvió a crecer la lesión con similares características en el mismo lugar, se le extirpó mediante exéresis elíptica y bisturí eléctrico. El análisis AP informó de la presencia de tejido tiroideo diferenciado en piel. Se trataba de una tumoración situada en dermis media y profunda constituida por estructuras foliculares de tamaños variables que contenían material coloide con vascularización periférica y revestidas por una hilera de células cúbicas con escaso número de mitosis. El examen inmunohistoquímico detectó expresión citoplasmática e intracoloidea de tiroglobulina, queratina 7 y 19 de manera intensa (Fig 1g, 1e y 1f, respectivamente). Con estos hallazgos la paciente nos fue remitida para valoración de metástasis de cáncer tiroideo no conocido previamente.

En la anamnesis la paciente se encontraba asintomática y carecía de antecedentes médicos de interés. En la exploración física se palpaba un bocio grado Ib con nódulo de $2-3 \mathrm{~cm}$ en LTD que ascendía bien con la deglución sin adenopatías palpables, siendo el resto normal. Pruebas complementarias: en análisis TSH 0,55 mU/L (0,35-5,5), T4l 1,23 ng/dL (0,8-1,76), T3l 3,19 $\mathrm{ng} / \mathrm{mL}(2,3-4,2)$, anticuerpos anti-TPO y anti-TG indetectables y TSI negativos que se han mantenido igual a lo largo del seguimiento. En la ecografía tiroidea se aprecia bocio multinodular, observándose en el LTD un nódulo dominante de 25 x 15 mm de ecogenicidad mixta con áreas hiper-hipoecoicas y calcificaciones groseras, y en su zona caudal se identificaron zonas mal definidas hipoecogénicas (Fig. 2), zona donde se realizó la PAAF y que fue informada de tumor folicular. Al mes se le realizó una tiroidectomía más linfadenectomía del compartimento central. La biopsia intraoperatoria fue informada de carcinoma papilar. El análisis AP de la pieza quirúrgica fue informado de carcinoma mixto papilar y folicular bien diferenciado (90\%) y sólido con un $10 \%$ poco diferenciado, parcialmente encapsulado con extensa infiltración de la cápsula y de los vasos de la misma. El tamaño de la porción encapsulada fue de $15 \mathrm{~mm}$ y de $30 \mathrm{~mm}$ el tumor completo. No se apreció extensión extratiroidea y los márgenes quirúrgicos estaban libres, siendo el más próximo el posterior a un mm. En el vaciado del compartimento central se obtuvieron 4 ganglios que resultaron libres de tumor.

Se le realizó estudio de extensión antes del tratamiento con radioyodo mediante estudio metabólico con 8,26 mCi de 18FDG, RM cerebral y pélvica y gammagrafía ósea que fue negativo. A los 70 días de la intervención se le administraron 104,7 mCi de I131 tras TSHrh. La TG sérica basal fue de $351 \mu \mathrm{g} / \mathrm{L}(1,6-60)$. TG estimulada 1788,7 


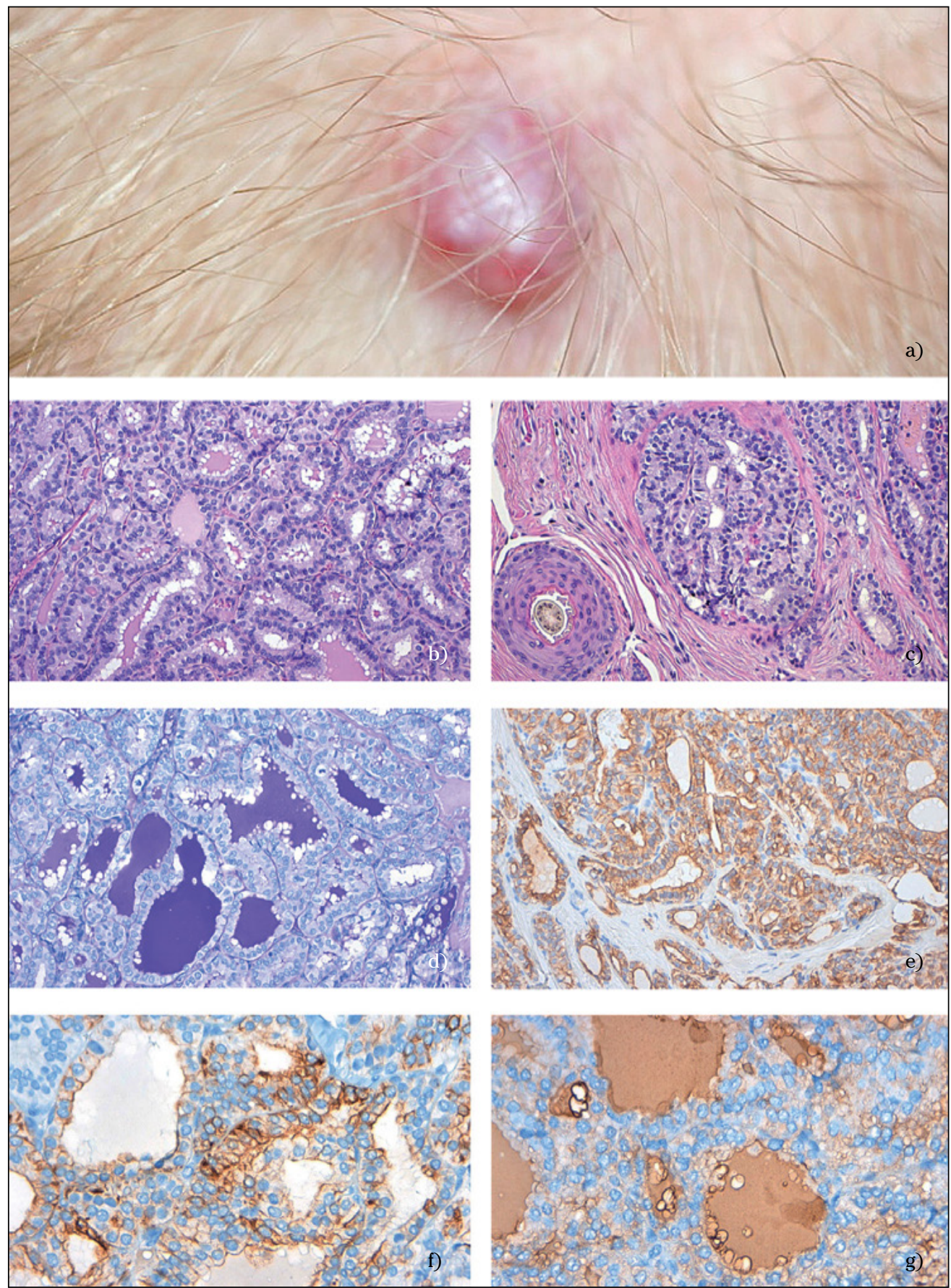

Figura 1. (a) Lesión papulosa eritemato-violácea dura de $6 \mathrm{~mm}$ en región parietooccipital. (b) Tejido tiroideo en dermis (H-E x40). (c) Tejido tiroideo próximo a folículo piloso (H-E x40). (d) Tinción PAS que tiñe intensamente el coloide de los folículos (PAS x40). (e) Inmunotinción con citoqueratina 7 positiva en células tumorales (IHQ x40). (f) Inmunotinción con citoqueratina 19 (IHQ x60). (g) Inmunotinción de tiroglobulina expresada en células tumorales y en coloide (IHQ x60). 
$\mu \mathrm{g} / \mathrm{L}(47-10000)$ con TSH basal de $117 \mathrm{mU} / \mathrm{L}(0,37-$ $4,7)$. En el RCT post-I131 se observaron restos post-tiroidectomía de localización en la cámara posterior izquierda, junto con afectación difusa de carácter miliar en ambos parénquimas pulmonares compatible con metástasis. Al mes de la ablación con radioyodo y en tratamiento con $100 \mu \mathrm{g}$ de levotiroxina presentó TG $37 \mu \mathrm{g} / \mathrm{L}(1,6-$ 60), TSH 1,96 mU/L y T4l 1,43 ng/dL, momento en que se le subió la dosis de levotiroxina a 125 $\mu$ g. En la evaluación a los 6 meses de la ablación con radioyodo la TG fue de 2,78 con TSH 0,03 y T4l 1,96, por lo que se le da otra dosis de radioyodo de $125 \mathrm{mCi}$; y 2 meses después se detecta $\mathrm{TG}<0,20 \mu \mathrm{g} / \mathrm{L}$ con TSH 0,04 mU/L y T4 1,6 ng/dL. La paciente será reevaluada en 4 meses mediante cuantificación de TG estimulada tras TSHrh y posterior RCT para confirmar curación.

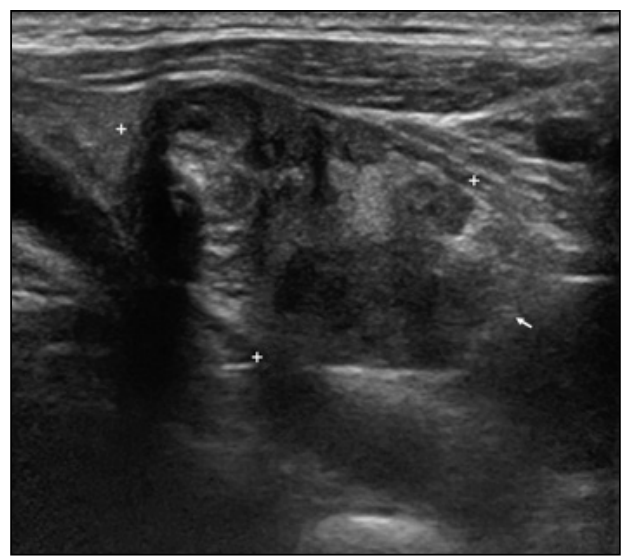

Figura 2. Ecografía tiroidea donde se aprecia en LTD un nódulo de 25 x 15 mm de ecogenicidad mixta con áreas hiper-hipoecoicas y calcificaciones groseras. En la zona caudal se señala zonas mal definidas hipoecogénicas donde se realizó PAAF.

\section{DISCUSIÓN}

La aparición de metástasis cutáneas en pacientes con cáncer es poco frecuente y llega a ser anecdótico en los casos de cáncer tiroideo. En una serie de 7.463 autopsias de pacientes que murieron por cáncer, sólo en el $2 \%$ de ellos se vieron metástasis en piel. En otro estudio donde se evaluaron 3.848 pacientes con cáncer metastásico, el 9\% tenían metástasis en piel, pero ninguno padecía neoplasia tiroidea ${ }^{4}$.En una serie descrita con 731 pacientes diagnosticados de cáncer papilar de tiroides, 91 tuvieron metástasis fuera de los ganglios linfáticos y sólo en 6 pacientes $(0,8 \%)$ se vieron en piel. Otra serie con 1.800 pacientes con cáncer papilar, 100 desarrollaron metástasis a distancia y sólo en un caso $(0,06 \%)$ se vio en piel $^{1}$.

Dahl y col en la Clínica Mayo han reunido en más de 35 años una de las series más largas de cáncer de tiroides con metástasis cutáneas, con un total de 43 casos (41\% con cáncer papilar, 28\% folicular, 15\% anaplásico y un 15\% medular), y sólo tienen un paciente similar al nuestro en el cual las metástasis cutáneas fueron el primer signo de presentación de la enfermedad ${ }^{1,2}$.

En 2005 se publicaron 4 casos de cáncer folicular de tiroides bien diferenciado con metástasis cutáneas ( 2 casos en cuero cabelludo, 1 en cuello y el otro en sacro), todos ellos en el contexto de una enfermedad tumoral diseminada ${ }^{5}$.

Posteriormente se han publicado casos de 6 pacientes en los que las metástasis cutáneas fueron el primer signo de la existencia de un cáncer diferenciado de tiroides, 5 se vieron en cuero cabelludo y en un caso en nariz. En todos los casos se vio en el estudio de la biopsia tejido tiroideo bien diferenciado con positividad a TG. De éstos 6, hay dos casos muy parecidos al nuestro que debutaron con metástasis en cuero cabelludo y en el estudio de extensión también se vieron en pulmón. Uno es un varón de 71 años con una metástasis en cuero cabelludo y una masa suprahiliar izquierda, con dos focos de carcinoma papilar variante folicular en la pieza quirúrgica sin ganglios linfáticos afectados ${ }^{1}$; y el otro caso es una mujer de 81 años con una metástasis parietal izquierda, con dos focos tumorales en LTD (el mayor correspondía a carcinoma folicular y el menor era papilar) con ganglios negativos y en el estudio de extensión se detectaron micrometástasis pulmonares como en nuestro caso ${ }^{3}$. El tercer caso es un varón de 73 años que debutó con una lesión en nariz y en el estudio se vio un carcinoma papilar localmente avanzado con invasión de tráquea y músculos 
paratraqueales con múltiples metástasis hepáticas y óseas ${ }^{6}$.

Otros dos casos son los únicos microcarcinomas diferenciados de tiroides diagnosticados por AP que debutan con metástasis cutáneas y en los que la ecografía tiroidea no fue capaz de detectar el primario. Uno es una mujer de 79 años que debutó con un nódulo en región occipital izquierda de más de 10 años de evolución con lesión lítica en zona craneal contigua al nódulo. En el LTI se detectó nódulo de 0,5 cm de carcinoma folicular, y en el RCT captaba el lecho quirúrgico y el área del hueso. Posteriormente los niveles de TG fueron subiendo, el paciente rechazó la realización de estudios complementarios y a los 3 años murió de enfermedad metastásica ${ }^{7}$. El otro caso es una mujer de 58 años con un nódulo en área occipitoparietal derecha desde hacía 8 años y en tiroides un microcarcinoma mixto papilar folicular de $0,7 \mathrm{~cm}$ con un ganglio positivo, rastreo negativo con $\mathrm{TG}<1$ y con buena evolución posterior al radioyodo ${ }^{7}$. El último de estos 6 casos, es una mujer de 82 años con una metástasis temporooccipital única al debut y cáncer folicular de tiroides con invasión vascular, en la que fue negativo el rastreo y los niveles de TG indetectables después de 3 años$^{8}$.

Las metástasis cutáneas de cáncer tiroideo son más frecuentes en cuero cabelludo y aparecen en forma de un nódulo violáceo o azul púrpura debido a la alta vascularización que presentan ${ }^{9}$. El diagnóstico anatomopatológico de las mismas mediante microscopía de luz es relativamente sencillo cuando se trata de cáncer diferenciado de tiroides con estructuras foliculares y material coloide como se vio en nuestro caso. Posteriormente se pudo confirmar por la expresión citoplasmática e intracoloidea de TG. Sin embargo, en otros casos en los que no se ve coloide y el tejido tampoco está suficientemente diferenciado a estructuras papilares y foliculares, es importante la utilización de técnicas inmunohistoquímicas para detectar TG, que tienen una sensibilidad cerca del 100\% para el cáncer folicular y del 95\% en el papilar. La expresión de TG es menor en los cánceres poco (o menos) diferenciados, siendo en los anaplásicos positiva en sólo un 40\% de los $\operatorname{casos}^{3}$. Sin embargo, hay tumores poco diferenciados que han perdido la capacidad de concentrar radioyodo por la pérdida de expresión del transportador de yodo NIS en la membrana celular, pero siguen produciendo TG y su medición es muy útil para detectar progresión tumoral.

A veces, las metástasis en piel son debidas a la transformación y desdiferenciación que sufren algunos cánceres de tiroides, con frecuencia ya tratados, a carcinomas poco diferenciados/anaplásicos de alto grado. Esto se vio en 2 pacientes de los 14 descritos en una serie con cáncer folicular metastásico a piel, y en los cuales la detección de TG fue negativa ${ }^{5}$. Otras veces, en el cáncer primario folicular/papilar se ven pequeños focos con menos diferenciación como se detectó en nuestra paciente y que parecen ser los responsables de la diseminación tumoral extratiroidea.

Por todo ello, es importante el diagnóstico temprano y correcto de las metástasis cutáneas de cáncer tiroideo, porque excepto en casos de enfermedad muy avanzada, el pronóstico suele ser bueno después de la terapia ablativa con radioyodo.

\section{BIBLIOGRAFÍA}

1. Somoza AD, Samaan S, Dhanda-Patil R, Mutasim DF. Cutaneous metastasis as the presenting sign of papillary thyroid carcinoma. J Cutan Pathol 2012; 40: 274-278.

2. Bruglia M, Palmonella G, Silvetti F y col. Skin and thigh muscle metastasis from papillary thyroid cancer. Singapore Med J 2009; 50: e61-e64.

3. Koller EA, Tourtelot JB, Pak HS, Cobb MW, MoAd JC, FLynN EA. Papillary and follicular thyroid carcinoma metastatic to the skin: a case report and review of the literature. Thyroid 1998; 8: 1045-1050.

4. Dahl PR, Brodland DG, Goellner JR, Hay ID. Thyroid carcinoma metastatic to the skin: a cutaneous manifestation of a widely disseminated malignancy. J Am Acad Dermatol 1997; 36: 531-537.

5. Quinn TR, Duncan LM, Zembowicz A, Faquin WC. Cutaneous metastases of follicular thyroid carcinoma: a report of four cases and a review of the literature. Am J Dermatopathol 2005; 27: 306-312. 
6. KHAN OA, Roses DF, Peck V. Papillary thyroid carcinoma metastatic to skin may herald aggressive disease. Endocr Pract 2010; 16: 446448.

7. Lissak B, Vannetzel JM, Gallouedec N, Berrod JL, Rieu M. Soltary skin metastasis as the presenting feature of differentiated thyroid microcarcinoma: report of two cases. J Endocrinol Invest 1995; 18: 813-816.
8. Karabekir H, Polat C, Aktepe F, Gocmen-Mas $\mathrm{N}$. Unusual scalp metastasis from follicular thyroid carcinoma. Saudi Med J 2011; 32: 849-851.

9. Kamangar F, McCalmont T, Shinkai K. Cutaneous metastasis of thyroid carcinoma mimicking Mondor disease. J Am Acad Dermatol 2012; 67: e79-e81. 